\title{
Diffusion of dust particles emitted from a fixed source
}

\author{
Khaled S. Al-Mashrafi \\ Section of Applied Sciences, Department of Human Resources Development, General Directorate of \\ Education in Eastern Region, Ministry of Education, Sur, Oman \\ E-mail: khaled2014om@gmail.com
}

Copyright () 2015 Khaled S. Al-Mashrafi. This is an open access article distributed under the Creative Commons Attribution License, which permits unrestricted use, distribution, and reproduction in any medium, provided the original work is properly cited.

\begin{abstract}
In this paper, we investigate the mathematical model for the diffusion of dust particles emitted from a fixed source. Mathematically, the time-dependent diffusion equation in the presence of a point source whose strength is dependent on time is solved. The solution in closed form for a source of general time dependence is obtained. A number of special cases, in which the source function of time is explicitly given and special values of the diffusion parameters are taken are examined in detail. The numerical calculations show the strong dependence of the concentration of dust on the speed of the wind, the source, and its position in the vertical direction. It is also found that the diffusion parameters play an important role in the spread of the dust particles in the atmosphere. When diffusion is present only in the vertical direction, it is found that for small times the dust spreads with a front that travels with the speed of the wind.
\end{abstract}

Keywords: Air Pollution; Atmospheric Diffusion Equation; Dust Concentration; Dust Diffusion; Wind Speed.

\section{Introduction}

The study of the mathematical model for the diffusion of dust particles emitted from a fixed source is relevant to some industrial and environmental applications [1 - 3]. Most industrial establishments have factories with chimneys through which the fumes escape into the atmosphere outside the factory. These fumes diffuse into the surroundings causing pollution and forming a health hazard [4 - 7]. In arid lands, strong winds can cause the dust from the ground to travel with wind and can cause danger to tarmac roads and also dirt in houses [8 - 11].

Small particles in the atmosphere are divided into 3 categories: (i) large ones (heavy) which are of diameter $0.5 \mathrm{~mm}$ or more, (ii) intermediate ones (medium) which are of diameter in the range 0.1-0.5 mm, and (iii) light ones (small) with the diameter less than $0.1 \mathrm{~mm}$. The dynamics of these particles depend on their size, the prevailing wind speed and the resulting turbulent eddies that disperse and diffuse the particles. An erodible bed is a type of surface, which has loose particles that can be removed easily by the wind. When wind blows over an erodible bed of small particles, the particles begin to move. The motion of such particles depends on a certain speed that is called threshold (critical) wind speed $U_{c}$. This speed depends on the size and surface roughness of the particles and logically it will be different for each particle. The particle will move from one place to another if the prevailing wind speed exceeded the critical speed for this particle.

For heavy particles, the influence of the gravitational force is strong and the threshold speed is large. So, these particles stay on the surface of the ground and their motion takes shape of rolling and/or sliding along the surface. This type of motion is called creep. The influence of gravity in the case of intermediate particles is smaller than that for heavy particles. So, their motion takes the form of bouncing along the surface. Such motion is known as saltation [12]. For the light particles, the critical velocity is small and the influence of gravity forces is not much. So, these particles can easily fly in the atmosphere and stay as suspension for long times before they fall down to the ground. This last family of particles includes natural dust, industrial fumes, and interstellar particles [13].

The diffusion of particles emitted from a certain source in the atmosphere is given by the atmospheric diffusion equation [14] 
$\frac{\partial C}{\partial t^{*}}+\mathbf{u} \cdot \nabla C=\nabla \cdot(\underline{\mathbf{D}}: \nabla C)-\mathbf{w} \cdot \nabla C \quad ; \quad \nabla \cdot \mathbf{u}=0$,

In which $C\left(\mathrm{r}, t^{*}\right)$ is the concentration of the dust particles after time $t^{*}, \mathbf{r}$ is position vector $\left(\mathrm{r}=x^{*} \mathrm{i}+y^{*} \mathrm{j}+z^{*} \mathrm{k}\right)$, $\mathrm{u}\left(\mathrm{r}, t^{*}\right)$ is the local velocity of the particles, $\mathrm{w}\left(\mathrm{r}, t^{*}\right)$ is the settling velocity, and $\underline{\mathrm{D}}(\mathrm{r})$ is the stress tensor and it is given in a Cartesian system of coordinates $O\left(x^{*}, y^{*}, z^{*}\right)$ by

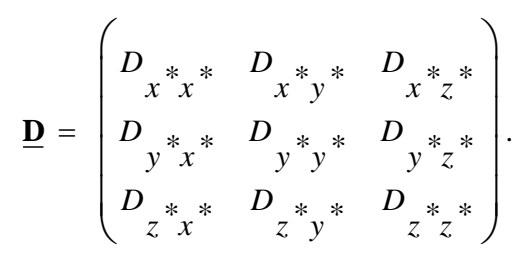

Where $O z^{*}$ vertically upwards and $O x^{*}$ and $O y^{*}$ are horizontal.

The settling velocity depends mainly on the size and on roughness of the particles. The stress tensor represents the local viscosity due to the friction between particles of the fluid. The left hand side of the equation (1) represents the rate of change of dust particles while the right hand side represents the flux. The second term on the left hand side represents the advection of the particles.

The spread of the dust particles has been studied theoretically and experimentally by using equation (1). Gillette and Goodwin [15] studied the steady state transport of sand-sized particles using the 1-D atmospheric diffusion equation ( $z$ - coordinate) in the case of moderate wind speeds. They supposed the absence of the advection term and the presence of non-zero vertical component of diffusion only. In their model, they assumed that the diffusion component varies with the vertical coordinate $z$, and the settling velocity is dependent only on the particle radius. They obtained an analytic solution for the concentration in closed form as a function of $z$. When they tested the applicability of their model to actual soil transport, they concluded that there is close agreement between them.

Hassan and Eltayeb [16] extended the Gillette and Goodwin model to find the concentration of the steady state 2-D diffusion equation. Considering Cartesian coordinates $O(x, y, z)$ with $O z$ vertical and $O x$ along the wind speed, they assumed that the wind move with the velocity $(U(z), 0,0)$, and the diffusion components are absent except the vertical one which was proportional to the vertical height $z$, the same as in the Gillette and Goodwin model. Their general result from this model was that the concentration is also dependent on the distance $x$ along the wind direction.

The study by Hassan and Eltayeb [17] examined the Gillette and Goodwin model in the presence of time variations as an initial -boundary-value-problem (IBVP) and found the solution in closed form for all time $t$. They used the atmospheric diffusion equation with homogeneity of concentration in the horizontal directions in the presence of the diffusion and gravitational forces. They assumed that the diffusion components are all zero except that in the vertical direction and it was linearly proportional to the height $\mathrm{z}$. They concluded that the concentration is very dependent on time, height, and settling velocity. When they tested the initial value problem as $t \rightarrow \infty$, they deduced the steady state of the Gillette and Goodwin model.

Sharan et al. [3] examined a steady-state model for low wind speeds. They assumed that the particles are very light and hence they neglected the gravitational force. They supposed that the air stream moved with a uniform velocity $U$ in the $x$-direction. Moreover, they assumed the presence of diffusion components in three coordinate directions and all of them are linearly proportional to the distance along the prevailing wind. They suggested a source of particles at ground level, as in the studies above. They compared their results with some observational data and they found reasonable agreement.

In the current study, we intend to extend the model by Sharan et al. [3] to include the time variation. This model applies for low wind speeds and is chosen because it is relevant to wind speeds in Oman most of the year. This model poses an initial-boundary-value problem for a linear partial differential equation of the second order. Here gravity is ignored but diffusion is potent. In section 2, we define the model. In section 3, we obtain the general solution of the problem in closed form. The solutions of some special cases with regard to the values of the diffusion parameters and the time dependence of the source, is examined in detail. In section 4, we present the solutions of the special cases graphically and discuss them in more detail as to their dependence on the parameters of diffusion. We also examine the solutions as the time increases indefinitely and compare the limiting solution with that of the steady state. In section 5, we make some concluding remarks. 


\section{Formulation of the model}

Define a Cartesian system of coordinates $O\left(x^{*}, y^{*}, z^{*}\right)$ in which $O z^{*}$ is vertically upwards, $O x^{*}$ and $O y^{*}$ are horizontal and the $x^{*}-$ axis is oriented in the direction of the wind speed. The concentration $C\left(x^{*}, y^{*}, z^{*}, t^{*}\right)$ of the dust particles in the time $t^{\prime}$ is governed by equation (1). We specify the velocity of dust particles and the settling velocity

$$
\mathbf{u}=(U, 0,0), \quad \mathbf{w}=\mathbf{0},
$$

Where $(U, 0,0)$ is the wind speed. Here we assume that the dust particles are very light.

We consider the diffusion tensor (2) has three non-zero components and are linear in the direction $x^{*}$, so that

$$
D_{x} * x^{*}=\alpha U x^{*}, D_{y} * y^{*}=\beta U x^{*}, D_{z} z^{*}=\gamma U x^{*},
$$

In which $\alpha, \beta$ and $\gamma$ are positive constants.

Using equations (3) and (4) to write equation (1) as

$$
\frac{\partial C}{\partial t^{\prime}}+U \frac{\partial C}{\partial x^{\prime}}=\frac{\partial}{\partial x^{\cdot}}\left(\alpha U x \cdot \frac{\partial C}{\partial x^{\prime}}\right)+\frac{\partial}{\partial y^{\prime}}\left(\beta U x \cdot \frac{\partial C}{\partial y^{\prime}}\right)+\frac{\partial}{\partial z}\left(\gamma U x \cdot \frac{\partial C}{\partial z^{\prime}}\right)
$$

Equation (5) represents the linearized three-dimensional unsteady atmospheric diffusion equation in the absence of gravitational forces. It describes the un-steady state diffusion of dust particles. We assume that system is due to a fixed source at $\left(0,0, h^{*}\right)$, where $h^{*} \geq 0$.

The relevant initial and boundary conditions are:

$$
\begin{aligned}
& C\left(x^{*}, y^{*}, z^{*}, t^{*}\right) \rightarrow 0 \quad \text { as } x^{*},\left|y^{*}\right|, z^{*} \rightarrow \infty, \\
& C\left(x^{*}, y^{*}, z^{*}, 0\right)=0 \\
& C\left(0, y^{*}, z^{*}, t^{*}\right)=\frac{\bar{Q}}{U} \delta\left(y^{*}\right) \delta\left(z^{*}-h^{*}\right) f\left(t^{*}\right) \text { with } f(0)=0 ; t^{*}>0, \\
& \frac{\partial C\left(x^{*}, y^{*}, 0, t^{*}\right)}{\partial z^{*}}=0,
\end{aligned}
$$

Where $\delta(s)$ is Dirac's delta function and condition (8) indicates that the source at $\left(0,0, h^{*}\right)$ has a strength $\bar{Q}$. The equation (5) subject to the conditions (6) - (9), poses an initial-boundary-value problem for a partial differential equation of second order. When $\alpha, \beta, \gamma>0$ and $x \geq 0$, the partial differential equation (5) is elliptic in nature. However if any two of the parameters $\alpha, \beta, \gamma$ vanish, the equation becomes parabolic.

\subsection{Solution of the general case}

If we define

$$
t=\alpha U t^{*}, x=x^{*}, z=\left(\frac{\alpha}{\gamma}\right)^{1 / 2} z^{*}, y=\left(\frac{\alpha}{\beta}\right)^{1 / 2} y^{*}, a=\frac{\alpha-1}{\alpha} \quad ; \alpha, \beta, \gamma \neq 0
$$

Then equation (5) becomes 
$x \frac{\partial^{2} C}{\partial x^{2}}+x \frac{\partial^{2} C}{\partial y^{2}}+x \frac{\partial^{2} C}{\partial z^{2}}+a \frac{\partial C}{\partial x}-\frac{\partial C}{\partial t}=0$,

(11)

With the initial and boundary conditions
(i) $C(x, y, z, 0)=0$;
$\left.\begin{array}{l}\text { (ii) } C(x, y, z, t) \rightarrow 0 \quad ; x,|y|, z \rightarrow \infty \\ \text { (iv) } C(0, y, z, t)=Q \delta(y) \delta(z-h) f(t), f(0)=0\end{array}\right\}$,
(iii) $\frac{\partial C(x, y, 0, t)}{\partial z}=0$;

Where

$$
h=\left(\frac{\alpha}{\gamma}\right)^{1 / 2} h^{*} \quad, \quad Q=\frac{\bar{Q}}{U}
$$

Now, we take the Fourier transform in $y$, Fourier Cosine transform in $z$, and Laplace transform in time of equation (11) and the conditions (12). Thus we obtain

$x \frac{d^{2} W}{d x^{2}}+a \frac{d W}{d x}-\left(x \lambda^{2}+x m^{2}+\omega\right) W=0$

Where

$W(x, \lambda, m, \omega)=\int_{0}^{\infty} \bar{C}(x, \lambda, m, t) e^{-\infty x} d t$,

$\bar{C}^{*}(x, \lambda, m, t)=\sqrt{\frac{2}{\pi}} \int_{0}^{\infty} \bar{C}(x, \lambda, z, t) \cos (m z) d z$,

$\bar{C}(x, \lambda, z, t)=\frac{1}{\sqrt{2 \pi}} \int_{-\infty}^{\infty} C(x, y, z, t) e^{\mathrm{i} \lambda y} d y \quad ; \quad \mathrm{i}=\sqrt{-1}$,

$W(x, \lambda, m, \omega)$ satisfies the conditions

$\left.\begin{array}{l}\text { (i) } W(x, \lambda, m, \omega) \rightarrow 0 \text { as } x \rightarrow \infty \\ \text { (ii) } W(0, \lambda, m, \omega)=\frac{Q}{2 \pi} \cos (m h) F(\omega)\end{array}\right\}$,

And we have defined

$F(\omega)=\int_{0}^{x} f(t) e^{-\omega t} d t$

The equation (14) can be reduced to Whittaker's equation

$\frac{d^{2} u}{d k^{2}}+\left(\frac{-1}{4}+\frac{\zeta}{k}+\frac{\frac{1}{4}-\eta^{2}}{k^{2}}\right) u=0$

By using the transformations 
$W(x, \lambda, m, \omega)=x^{-a / 2} u(x, \lambda, m, \omega)$.

$\zeta=\frac{-\omega}{2 s}, \quad \eta=\frac{a-1}{2}, \quad s^{2}=\lambda^{2}+m^{2}, \quad k=2 s x$,

The equation (20) has the general solution [18]

$u(k, \lambda, m, \omega)=e^{-k / 2} k^{a / 2}\left[A M\left(\frac{a}{2}+\frac{\omega}{2 s}, a, k\right)+B U\left(\frac{a}{2}+\frac{\omega}{2 s}, a, k\right)\right]$

In which $A$ and $B$ are constants and $M(a, b, x), U(a, b, x)$ are the Confluent hypergeometric functions of first and second kind, respectively [19].

Then using (22) - (22) to express $W(x, \lambda, m, \omega)$ in the general form

$W(x, \lambda, m, \omega)=e^{-\mu}(2 s)^{\omega-2}\left\{A M\left(\frac{a}{2}+\frac{\omega}{2 s}, a, 2 s x\right)+B U\left(\frac{a}{2}+\frac{\omega}{2 s}, a, 2 s x\right)\right\}$.

The application of the conditions (18) and using the properties of the Confluent hypergeometric functions [19] when $x \rightarrow 0$ and $x \rightarrow \infty$ lead to

$A=0$,

$B=\frac{Q}{2 \pi} \cos (m h) F(\omega) \frac{\Gamma\left(1-\frac{a}{2}+\frac{\omega}{2 s}\right)}{\Gamma(1-a)}(2 s)^{-\omega / 2}$,

Where $\Gamma(a)$ is Gamma function.

The solution of equation (24) then can be written in the form

$W(x, \lambda, m, \omega)=\frac{Q}{2 \pi \Gamma(1-a)} \cos (m h) F(\omega) \Gamma\left(1-\frac{a}{2}+\frac{\omega}{2 s}\right) e^{-s x} U\left(\frac{a}{2}+\frac{\omega}{2 s}, a, 2 s x\right)$.

The next step is taking the inverse Fourier transform with respect to $y$ and inverse Fourier Cosine with respect to $z$ and the inverse Laplace transform with respect to time for the expression (27). After some algebraic simplifications, we obtain

$C(x, y, z, t)=\frac{Q}{8 \mathrm{i} \pi^{2} \Gamma(1-a)} \int_{c-\mathrm{i} \infty}^{c+\mathrm{i} \infty} F(\omega) \phi(x, y, z, \omega) e^{\omega t} d \omega ; c>0$,

Where, all the poles $\omega_{\mathrm{i}}$ of $F(\omega) \phi(x, y, z, \omega)$ lie in $\operatorname{Re}(\omega)<c$, and the function $\phi(x, y, z, \omega)$ is given by

$\phi=\int_{0}^{\infty}\left\{J_{0}\left(r \sqrt{y^{2}+(z+h)^{2}}\right)+J_{0}\left(r \sqrt{y^{2}+(z-h)^{2}}\right)\right\} \Gamma\left(1-\frac{a}{2}+\frac{\omega}{2 s}\right) e^{-s x} U\left(\frac{a}{2}+\frac{\omega}{2 s}, a, 2 s x\right) r d r$.

In which $J_{0}(x)$ is Bessel function of the first kind of order 0 , and we have defined the following circular transformation

$$
\lambda=r \cos (\theta) \quad, \quad m=r \sin (\theta),
$$

Where the Jacobian of this transformation is given by 


$$
J=\left|\begin{array}{cc}
\lambda_{r} & \lambda_{\theta} \\
m_{r} & m_{\theta}
\end{array}\right|=r .
$$

The expressions (28) and (29) give the solution for the concentration in the $(x, y, z)$ plane at all times. The solution (28) has a complicated dependence on the variables of the system. However, we can get some understanding of the influence of diffusion parameters in all directions by studying some special cases of the parameters $\{\alpha, \beta, \gamma\}$. In this paper, we consider the case $\alpha=\beta=0 ; \gamma \neq 0$.

\subsection{Solution in the case of vertical diffusion $(\alpha=\beta=0, \gamma \neq 0)$}

This case happens when the vertical diffusion only is present, and both longitudinal (along $x$ ) and latitudinal (along $y$ ) diffusions are absent. Thus, the solution is given by the expression

$$
C(x, z, t)=\frac{Q f(t-x) H(t-x)}{2 \sqrt{2 \pi} x}\left[\exp \left\{\frac{-(h+z)^{2}}{2 x^{2}}\right\}+\exp \left\{\frac{-(h-z)^{2}}{2 x^{2}}\right\}\right],
$$

Where $t, x, z, h, Q$ are given in the equations (10) and (13) and $H(x)$ is the Heaviside function.

Expression (32) represents the general solution for a general source $f(t)$ in closed form. This expression specifies the concentration at every point $(x, z, t)$ of the domain. We note that the time dependence appears only in the amplitude of the concentration and is absent in the exponential dependence. We also note that the presence of the Heaviside unit function in the amplitude of the solution represents the discontinuity in the solution across the straight line $x=t$ in the $(x, t)$ plane. In section 4 below, we will illustrate this solution by three examples of the source function $f(t)$.

\section{Discussion}

Expression (32) gives the general solution of the concentration for a source with time-dependence $f(t)$ when vertical diffusion $\left(D_{w}\right)$ is present, and both longitudinal and latitudinal diffusions, $D_{w}$ and $D_{v}$, are absent. Here we specify the solution of the concentration of dust particles using three examples of function $f(t)$, which are (i) Heaviside function $H(t)$, and two exponential functions (ii) $1-e^{-\lambda}, \lambda>0$ and (iii) $1-e^{-t^{-2}}$. The main difference between these examples of function is that the first one has a jump from 0 to 1 at $t=0$, and the others start from 0 and then increase continuously until they reach 1. Our aim for choosing these specific examples is that we want to see the effect of the strength of the source as time varies. In the case of the first example, the strength of the source doesn't depend on the time after $t=0$. While in the second and third examples, the strength of the source depends on the time $t$.

\subsection{The source function with $f(t)=H(t)$}

The general expression of the solution (32) can be written in the form

$$
C(x, z, t)=\left\{\begin{array}{cc}
\frac{Q}{2 \sqrt{2 \pi} x}\left[\exp \left\{\frac{-(h+z)^{2}}{2 x^{2}}\right\}+\exp \left\{\frac{-(h-z)^{2}}{2 x^{2}}\right\}\right] & , x<t \\
0 & , x>t
\end{array} .\right.
$$

The contours of the solution (33) in the $(x, z)$ plane are illustrated in figures 1 and 2 for different values of time $t$. We observe that the solution has a discontinuity at $x=t$ for small $t$. Noting the transformation (10) we see that the line $x-t=$ constant corresponds to $x^{*}-U t^{*}=$ constant. So that on this characteristic $d x^{*} / d t^{*}=U$, i.e. the concentration of particles travels with a speed $U$ away from the source. This is, illustrated for two sample values of $h(=0.0,5.0)$ in figures 1 and 2, respectively. The case $h=0.0$ corresponds to pollution from a car exhaust while that at $h=5.0$ is representative of pollution due to an industrial chimney. In this case, the source of pollutant doesn't depend on the time. For $x<t$, the concentration decreases away from the source and spreads further from the source as $t$ increases. For $x>t$, there is no pollutant. This situation applies for all values of the height of the source above ground level. For small values of the time $t$, the proximity of the front at $x=t$ to the source causes the pollution to diffuse upwards, (figures 1,2(a), (b), (c)). For large values of time $t$, the discontinuity at $x=t$ has no effect on the distribution of the pollutant. This is because the source is not strong enough to force the pollution far away from the origin. As a result the 
pollution concentration is reduced near the source. We notice that the height of the source above ground level affects the distribution of dust on the ground. When the source is situated on the ground, the pollution on the ground is strong but as the height increases, the pollution spreads over a larger area both horizontally and vertically. When $t \rightarrow \infty$, the distribution of dust approaches the steady state solution, as can be seen from the figures 1(f) and 2(f).

Figures 3 and 4 show the sketch of the solution (33) in the $(x, z, \bar{C})$ space for two values of the source height $h$ and fixed time $t$. The surface of the concentration here is drawn in 3-dimensions. The characteristic line $x=t$ appears in the figure. We deduce from figures 3 and 4 that the front moves with the speed of the wind.
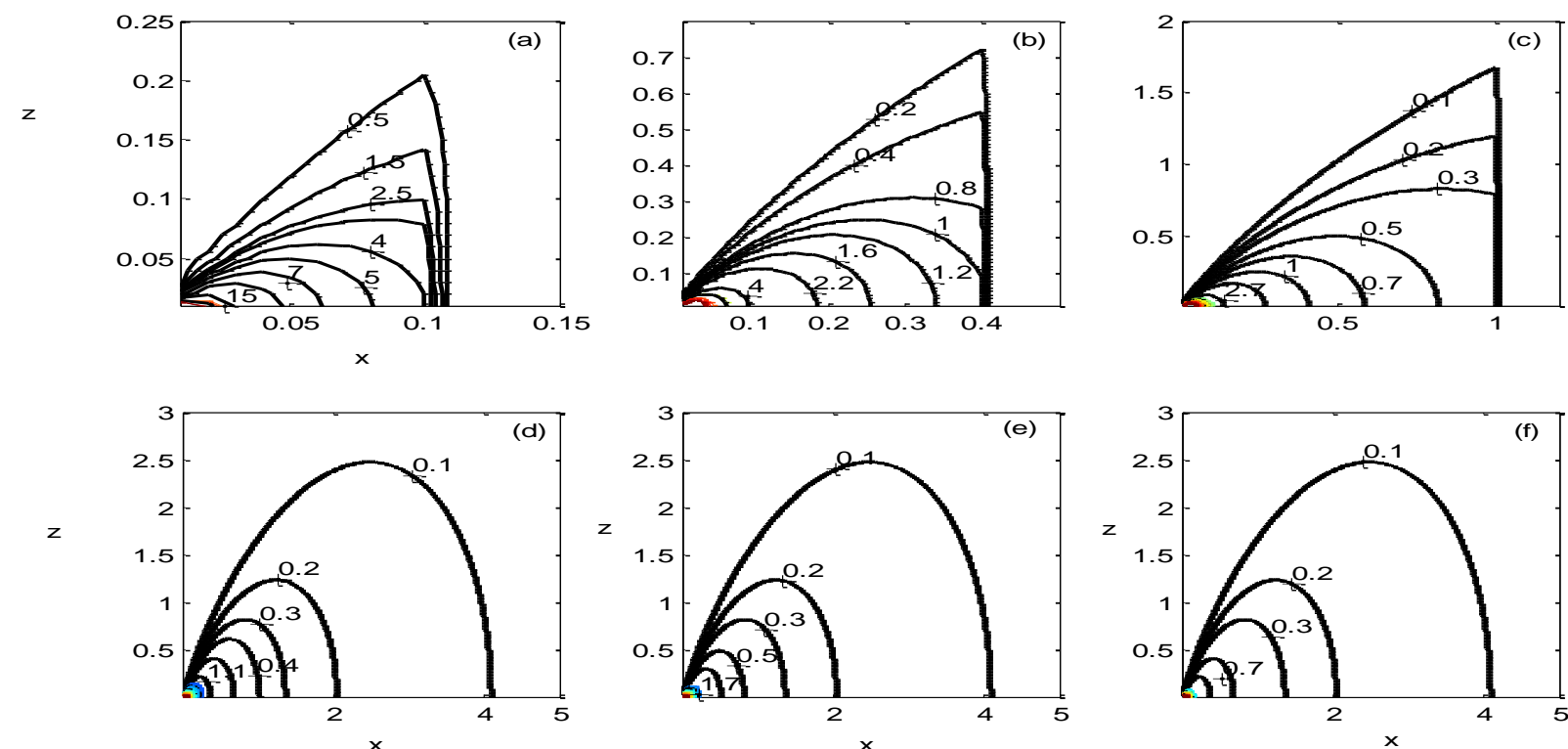

Fig. 1: The Isolines of the Concentration $\bar{C}(x, z, t)=C(x, z, t) / Q$ in the $(x, z)$ Plane when $f(t)=H(t)$ and $h=0.0$, for Different Values of the Time: (A) $t=0.1$, (B) $t=0.4$, (C) $t=1.0$, (D) $t=5.0$, (E) $t=7.5$, and (F) $t=10.0$. Note the Precipitation of the Pollutant on the Ground as the Time Increases. Note the Position of the Characteristic $x=t$ as $t$ Is Increased. the Scales are Different for the Sake of Clarity.
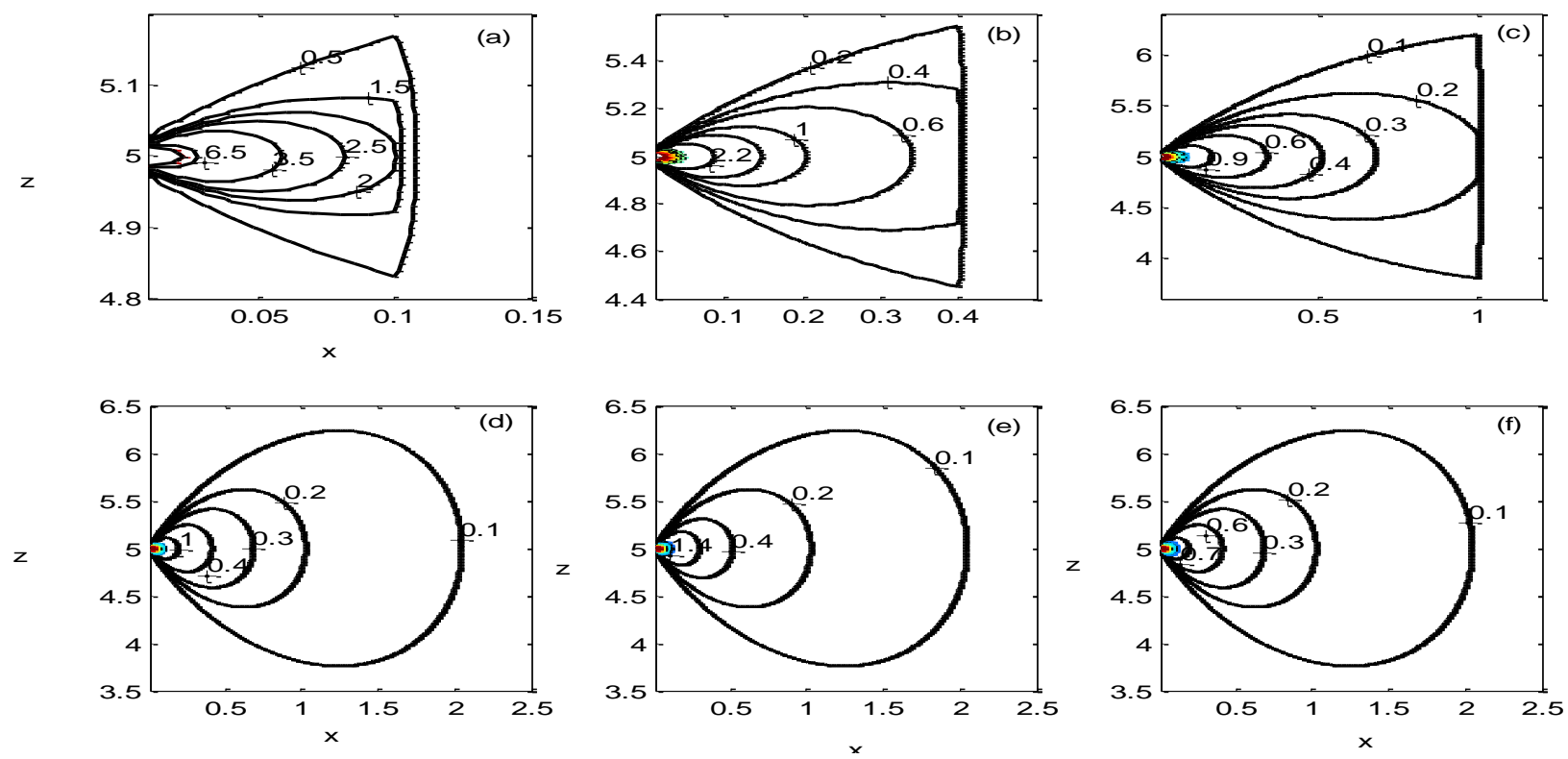

Fig. 2: The Isolines of the Concentration $\bar{C}(x, z, t)=C(x, z, t) / Q$ in the $(x, z)$ Plane when $f(t)=H(t)$ and $h=5.0$, for Different Values of the Time: (A) $t=0.1$, (B) $t=0.4$, (C) $t=1.0$, (D) $t=5.0$, (E) $t=7.5$, and (F) $t=10.0$. Compare Figures 1 and 2 to Notice the Influence Of Increasing the Height of An Industrial Chimney. the Scales are Different for the Sake of Clarity. 


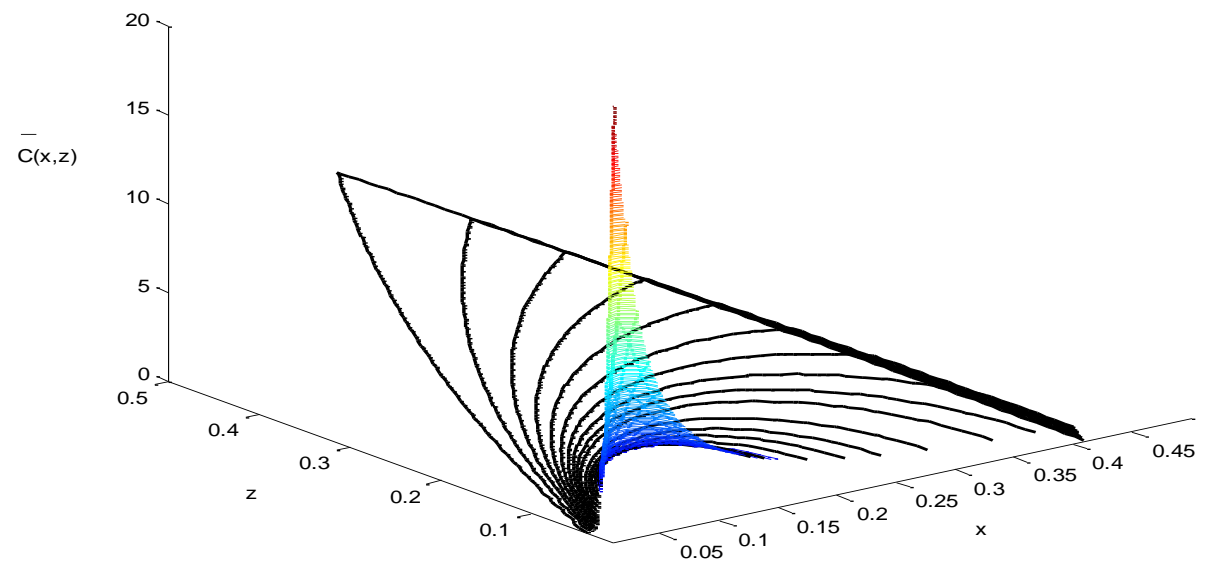

Fig. 3: The Profiles of the Concentration $\bar{C}(x, z, t)=C(x, z, t) / Q$ in the $(x, z, \bar{C})$ Space when $f(t)=H(t)$ and $h=0.0$, for $t=0.4$. Note the Characteristic Line $x=0.4$. Compare with Figure 1(B).

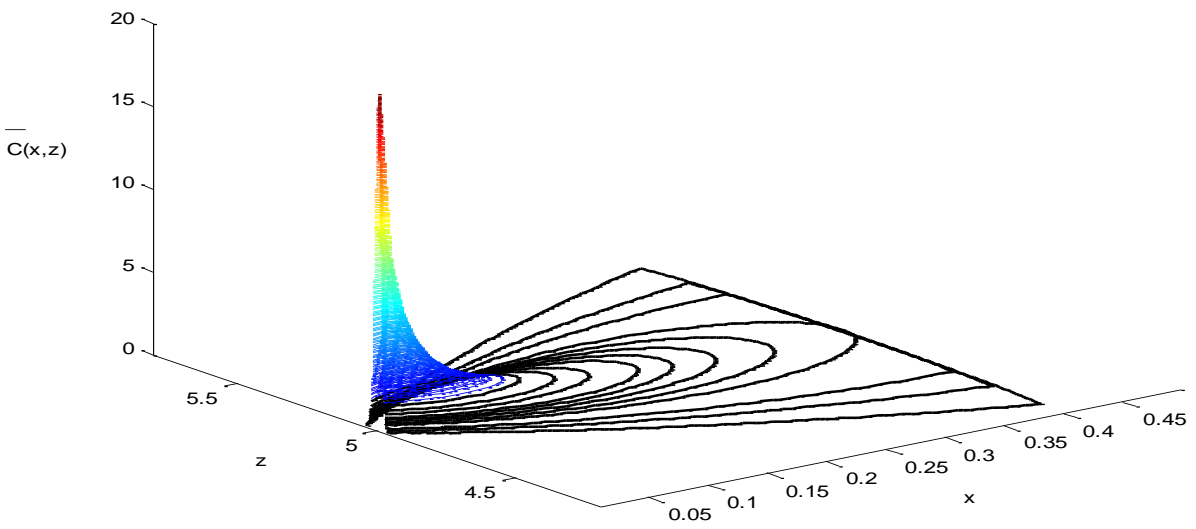

Fig. 4: The Profiles of the Concentration $\bar{C}(x, z, t)=C(x, z, t) / Q$ in the $(x, z, \bar{C})$ Space When $f(t)=H(t)$ and $h=5.0$, for $t=0.4$. Compare with Figure 2(B). Note the Pollutant Concentrated Very Close to the Source.

\subsection{The source function with $f(t)=1-e^{-\lambda t}, \quad \lambda>0$}

The solution (32) becomes

$C(x, z, t)=\left\{\begin{array}{c}\frac{Q}{2 \sqrt{2 \pi} x}\left(1-e^{-\lambda(t-x)}\right)\left[\exp \left\{\frac{-(h+z)^{2}}{2 x^{2}}\right\}+\exp \left\{\frac{-(h-z)^{2}}{2 x^{2}}\right\}\right], x<t . \\ 0 \quad x>t\end{array}\right.$

The contours of the solution (34) in the $(x, z)$ plane are illustrated for different values of the time $t$ and decay factor $\lambda$ in figures 5-8. We note that the strength of the source in this case is an exponential function of the time, and hence the strength of the source depends on the coefficient of decay $\lambda$ as well as on the time. When this coefficient is small, then the strength of the source is weak for small times. Figures 5 and 6 illustrate the profiles of the solution for a fixed value of $\lambda=10.0$ and different values of the time $t$ with two different heights of the source. We observe that there is no discontinuity in the distribution of the pollutant in the $(x, z)$ plane whatever the values of the time and height. This is due to the fact that the source is very weak for small times and hence the emission of pollution is weak and this gives the discontinuity at $x=t$, which travels with the speed of the wind, time to travel further than the front of the pollution. Thus the line $x=t$ is present but the pollution is behind the line and there is no actual discontinuity in the distribution of the pollutant.

As we have seen in case (i), the height of the source above ground level affects the spread of pollutant in the space. This state happens here also. Figures 5 and 6 illustrate the relation between the height of the source and the area that is covered by the pollutants in the space. For large values of the time $t$, the distribution of the pollutant converges to the steady state solution (figures 5 and 6(f)). 
Figures 7 and 8 show the profiles of the concentration of pollutants in the space for a fixed value of the time $t=1.0$ and different values of the decay factor $\lambda$ for two different values of the height of the source. For small values of $\lambda$, the strength of the source is weak and its ability to push the pollutant far away from the origin is not strong. Hence, the pollutant cannot spread far away from the source and so the characteristic line at $x=1$ is far ahead of the pollutant (figure 7,8 $(a, b)$ ). When we choose a large value of the decay factor $\lambda$, the strength of the source of the pollutant is increased. Then the particles diffuse in large areas in the atmosphere, and this resulted in the appearance of the characteristic line $x=1$ (figure $7,8(\mathrm{c}, \mathrm{d})$ ). Furthermore, when $\lambda \rightarrow \infty$, the spread of the particles approaches the distribution of particles in case (i) (see figures 7(d), 8(d) and compare them with figures 1(c) and 2(c), respectively). These situations happened due to the relation of the Heaviside function in case (i) and exponential function in case (ii) for large values of $\lambda$.

The height of the source above ground level plays an important role in the distribution of the pollutant in the atmosphere. Whenever the source of pollutant is raised, the pollution spreads both upwards and downwards as well as horizontally and hence can cover a wider area than that at ground level.
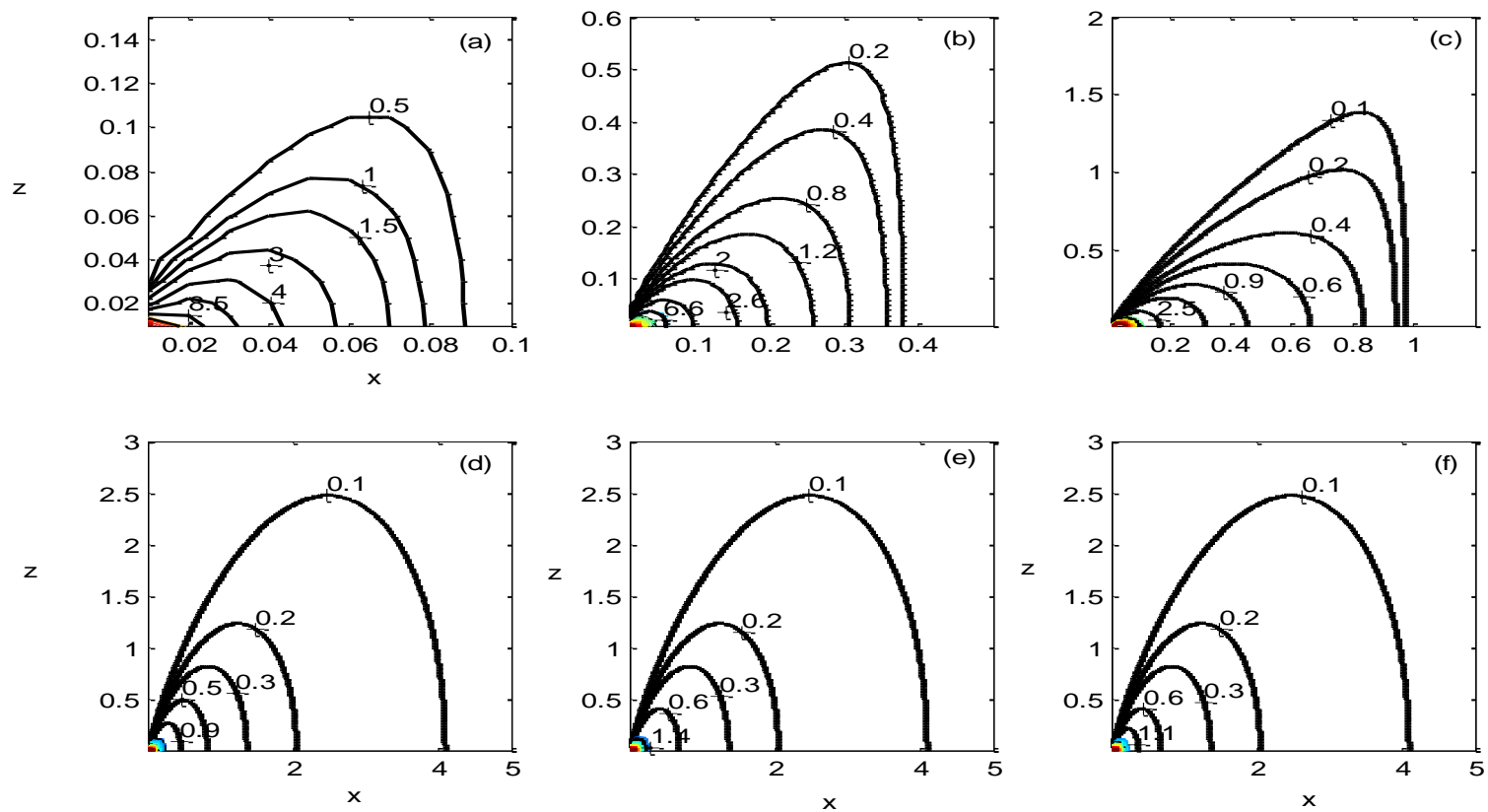

Fig. 5: The Isolines of the Concentration $\bar{C}(x, z, t)$ in the $(x, z)$ Plane when $f(t)=1-e^{-\lambda t}$ for $h=0.0$, and $\lambda=10.0$ and the Time $t$ Takes the Values 0.1,0.4,1.0,5.0,7.5, and 10.0 in (A), (B), (C), (D), (E) and (F), Respectively. The Scales are Different for the Sake of Clarity.
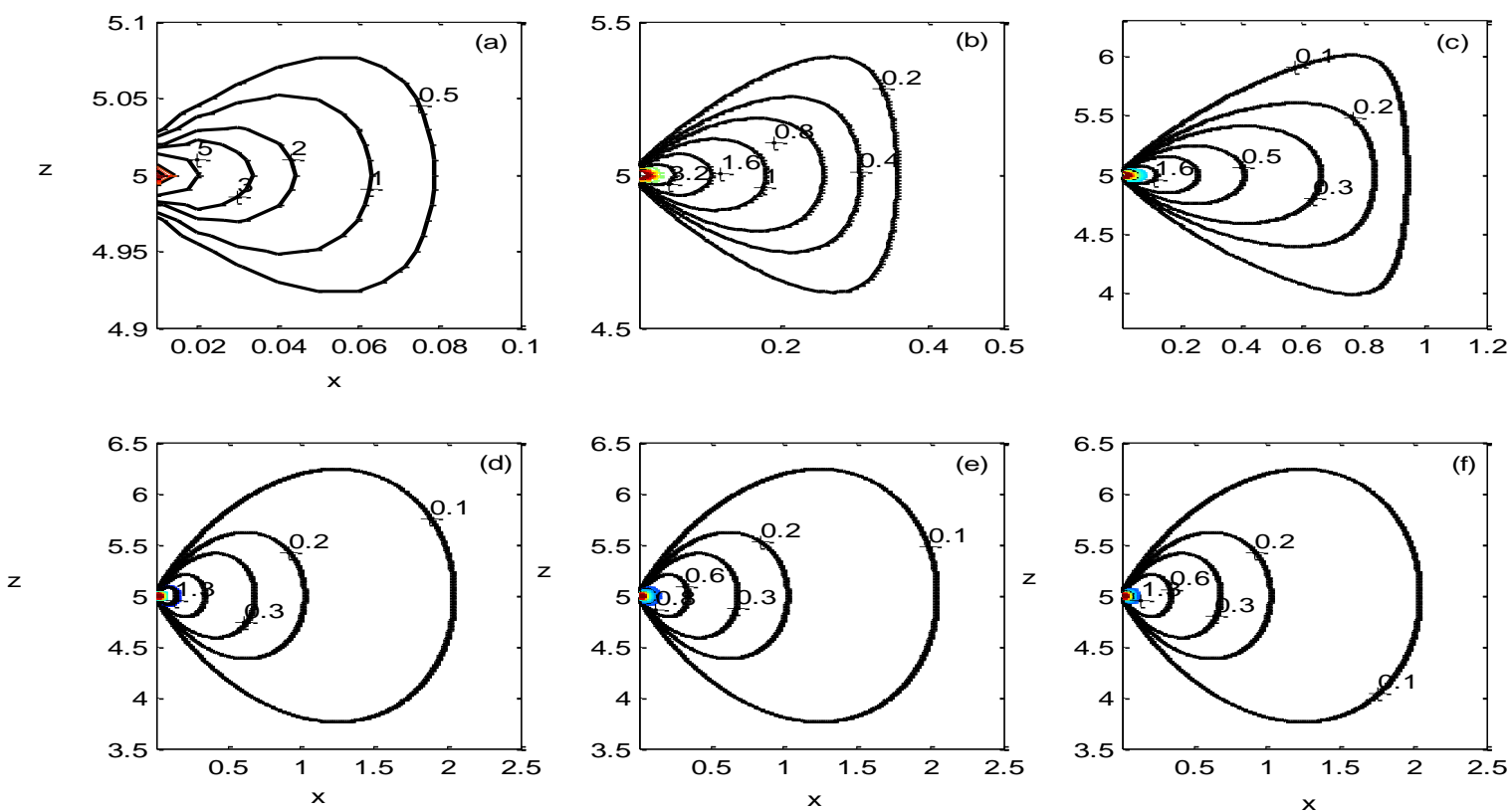
Fig. 6: The Isolines of the Concentration $\bar{C}(x, z, t)$ in the $(x, z)$ Plane when $f(t)=1-e^{-\lambda t}$ for $h=5.0$, and $\lambda=10.0$ and the Time $t$ Takes the Values $0.1,0.4,1.0,5.0,7.5$, and 10.0 in (A), (B), (C), (D), (E) and (F), Respectively. Compare Figures 5 and 6 to Notice the Influence of Increasing the Height of an Industrial Chimney.
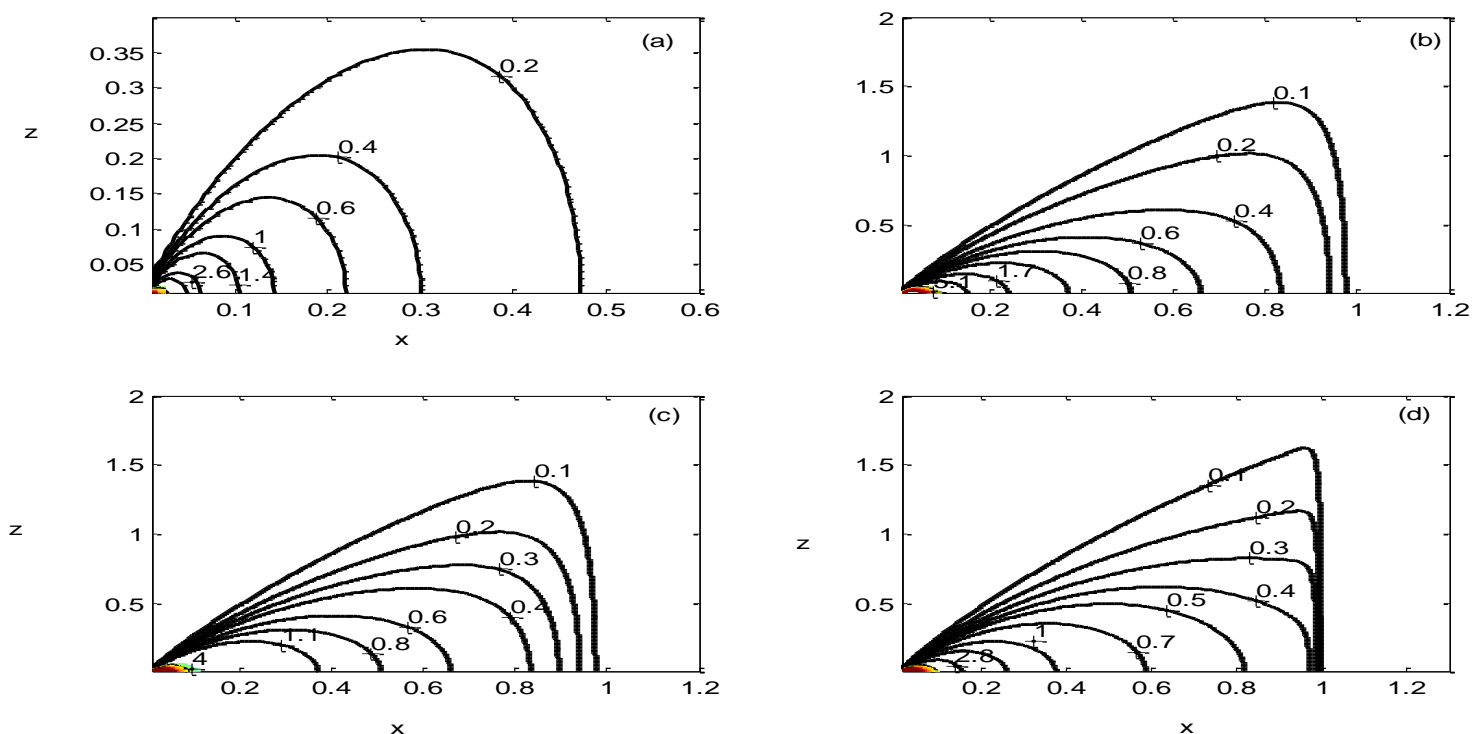

Fig. 7: The Isolines of the Concentration $\stackrel{\times}{C}(x, z, t)$ in the $(x, z)$ Plane when $f(t)=1-e^{-\lambda t}$ for $h=0.0$, and $t=1.0$ and the Factor $\lambda$ Takes the Values $0.5,1.0,10.0$, and 100.0 in (A), (B), (C) and (D), Respectively. Note the Increasing of the Coefficient of Decay.
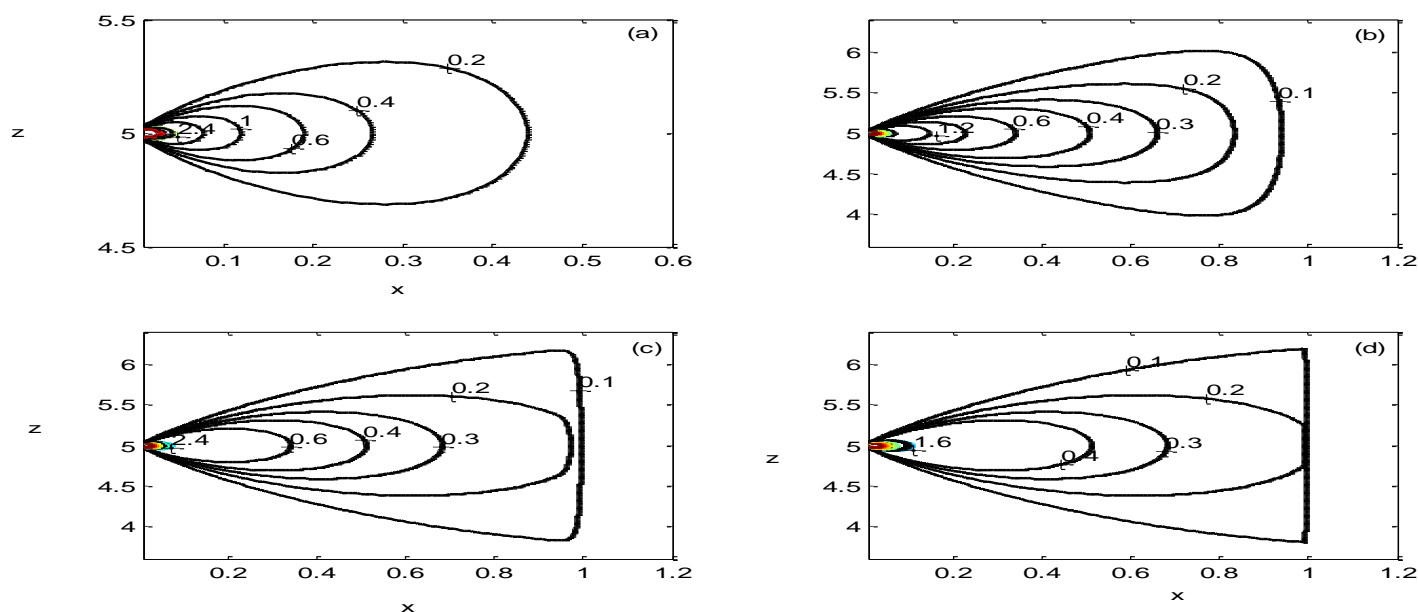

Fig. 8: The Isolines of the Concentration $\bar{C}(x, z, t)$ in the $(x, z)$ Plane when $f(t)=1-e^{-\lambda t}$ for $h=5.0$, and $t=1.0$ and the Factor $\lambda$ Takes the Values 1.0,10.0,100.0, and 1000.0 in (A), (B), (C) and (D), Respectively Compare with Figure 3(C).

\subsection{The source function with $f(t)=1-e^{-t^{2}}$}

The solution (32) becomes

$C(x, z, t)=\left\{\begin{array}{l}\frac{Q}{2 \sqrt{2 \pi} x}\left(1-e^{-(t-x)^{2}}\right)\left[\exp \left\{\frac{-(h+z)^{2}}{2 x^{2}}\right\}+\exp \left\{\frac{-(h-z)^{2}}{2 x^{2}}\right\}\right], \\ 0 \quad x<t . \\ , x>t\end{array}\right.$.

The contours of the solution (35) in the $(x, z)$ plane are illustrated for different values of the time $t$ in figures 9 and 10 . We note here that the source is very weak when $t$ is small. This has the effect that the concentration of the pollutant spreads with slow speed (i.e. much less than $U$ ) and hence the characteristic travels with a faster speed so that there is no discontinuity in the concentration of the pollutant. The pollution decreases away from the source. 
For large values of the time $t$, the source is still weak and unable to push the pollutants away from it. Then the characteristic doesn't appear in the figures. In general, the behaviour of the solution in this case is very similar to case (ii) except that the distribution decreases more rapidly as the distance from the source is increased.

The influence of the height of the source on the distribution is the same as that discussed in cases (i) and (ii). The distribution of the pollutants in the atmosphere converges to the steady state solution when $t \rightarrow \infty$.

Comparison between the three cases of the function $f(t)$ shows that the dependence of the source on the time has an influence on the distribution of the pollutant in the space. This applies to all values of the height (figures $1-10$ ).

The solution of the case (i) in which the source function is 1 for all $t>0$, is identical with the limiting case (ii) when the parameter $\lambda \rightarrow \infty$ for all $t \neq 0$. The solution of case (iii) also approaches that of case (i) as $t \rightarrow \infty$. For small and moderate values of the time $t$, the concentration of pollution at a general point $(x, z)$ is different in the three cases. This is because the strength of the source at these times is different for the three cases. The uniform strength of the source for case (i) resulted in the appearance of the discontinuity after small $t$ while the disappearance of the discontinuity in cases (ii) and (iii) is due to the weak sources so that the pollution is unable to reach the discontinuity.
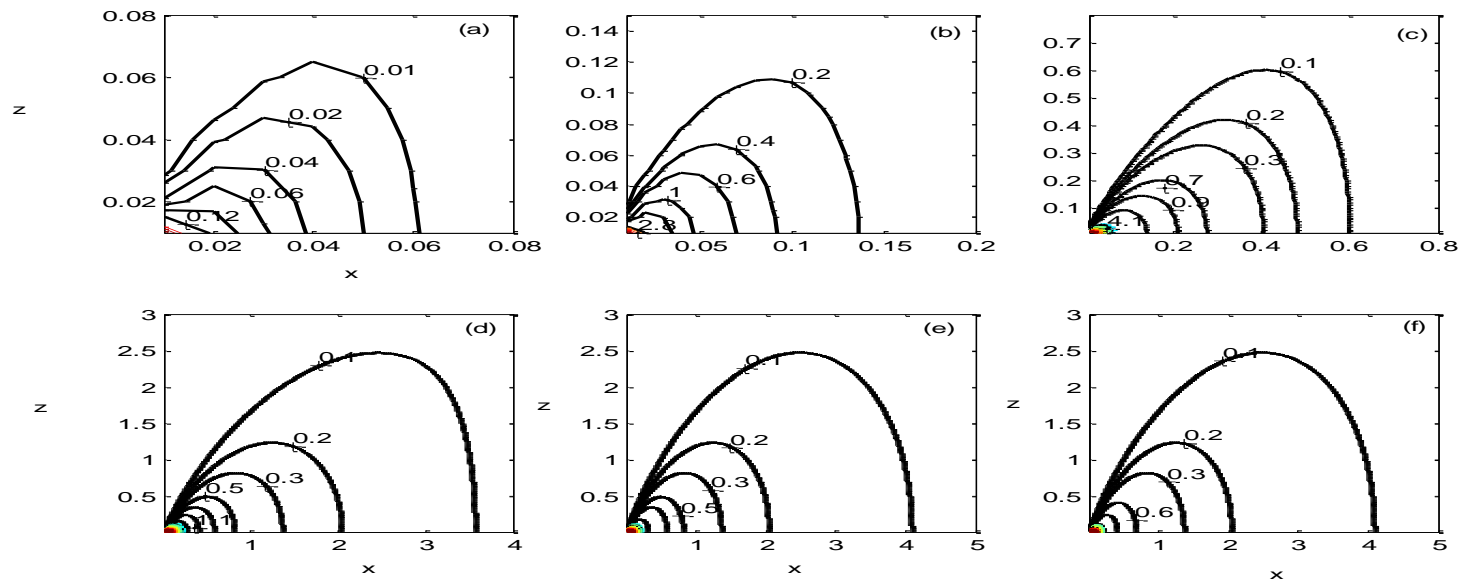

Fig. 9: The Isolines of the Concentration $\bar{C}(x, z, t)$ in the $(x, z)$ Plane when $f(t)=1-e^{-t^{2}}$ and $h=0.0$ for Different Values of the Time: (A) $t=0.1$, (B) $t=0.4$, (C) $t=1.0$, (D) $t=5.0$, (E) $t=7.5$, and (F) $t=10.0$.
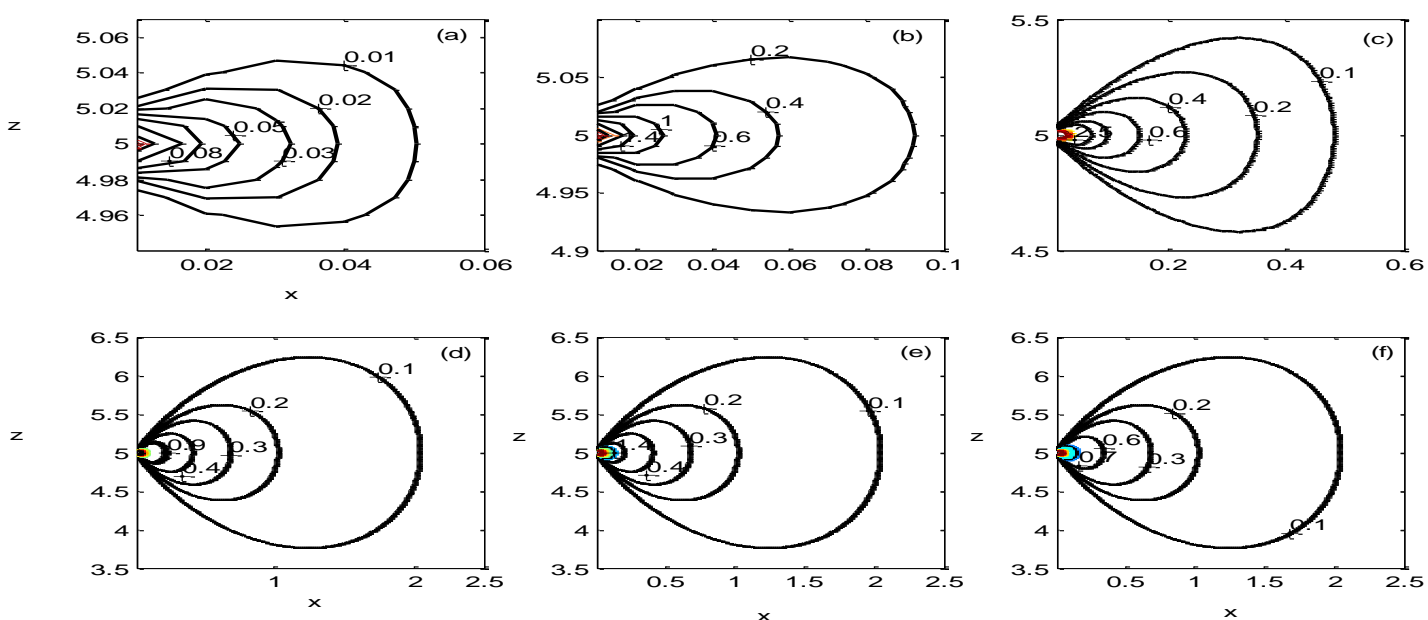

Fig. 10: The Isolines of the Concentration $\bar{C}(x, z, t)$ in the $(x, z)$ Plane when $f(t)=1-e^{-t^{2}}$ and $h=5.0$ for Different Values of the Time: (A) $t=0.1$, (B) $t=0.4$, (C) $t=1.0$, (D) $t=5.0$, (E) $t=7.5$, and (F) $t=10.0$. Note the Rise of Dust as $t$ Increases.

For the limiting cases, we examine the solutions (34) and (35) for large values of the time. For large values of the time, they become

$\left.C(x, z, t)\right|_{t \rightarrow \infty}=\frac{Q}{2 \sqrt{2 \pi} x}\left[\exp \left\{\frac{-(h+z)^{2}}{2 x^{2}}\right\}+\exp \left\{\frac{-(h-z)^{2}}{2 x^{2}}\right\}\right]$

Which is the same as solution (33). This means that the solutions in the cases (ii) and (iii) are uniformly convergent to the solution in the case of the function $f(t)=H(t)$ when $t \rightarrow \infty$. Furthermore, for the limit $\lambda \rightarrow \infty$, the solution (34) 
reduced to (33). We can say that the solution of this problem in the case of the function $f(t)=1-e^{-x}$ is uniformly convergent to the solution in the case of the function $f(t)=H(t)$ when $\lambda \rightarrow \infty$ or $t \rightarrow \infty$ or both. The solutions of the three specific examples above showed that the solution tends to the same value as $t \rightarrow \infty$.

\section{Conclusion}

A mathematical model for the diffusion of dust particles from a fixed source has been studied. The distribution of such particles in the air is given by an initial boundary value problem (IBVP) of the atmospheric diffusion equation. The diffusion of the particles at every point is dependent on the locality of that point. We have assumed that diffusion has three components, which depend on the distance along the wind direction. Such a model is relevant to small wind speeds. An analytical expression of the concentration at every point of space is obtained in closed form. However, the general solution is very complicated and depends on the right choice of contour for evaluation.

We have therefore chosen to consider a special case where explicit solutions are obtained. In this case, diffusion in the both longitudinal and latitudinal directions was neglected. The solution is obtained explicitly for all values of time, distance and height. The dependence of the distribution of dust on the time variation of the source is investigated for three different functions. The extent of spread of dust depends on the strength of the source. When the source is strong for small times, the solution shows a discontinuity.

A general point introduced by this model was that the dependence of the source on the time affects the way the pollutant emitted from a fixed source is distributed in the atmosphere. Another general result obtained is that in all the special cases considered here the asymptotic behaviour of the solutions for large times approaches the steady state solution obtained previously by Sharan et al. [3]. The case in which the diffusion in horizontal direction is present, will be studied in the next paper.

\section{Acknowledgements}

The author would like to express most sincere appreciation and gratitude to Prof. Ibrahim A. Eltayeb (College of Science, Sultan Qaboos University) for his cooperation and support.

\section{References}

[1] El-Baz, F., Eltayeb, I.A. and Hassan, M.H.A., "Sand Transport and Desertification in Arid and Semi-Arid Lands", World Scientific Publishing Co., Singapore (1990).

[2] Hassan, M.H.A. and Eltayeb, I.A., "Suspension transport of wind eroded sand particles", Geophys. J. Internet. , 104, (1991b), pp: 147-152. http://dx.doi.org/10.1111/j.1365-246X.1991.tb02500.x.

[3] Sharan M., Singh M.P., and Yadav, A.K., "Mathematical model for atmospheric dispersion in low winds with eddy diffusivities as linear functions of downwind distance", Atmospheric Environment, 30, (1996), pp: 1137-1145. http://dx.doi.org/10.1016/1352-2310(95)00368-1.

[4] Roberts, O.F.T., "The theoretical scattering of smoke in a turbulent atmosphere", Proc. Roy. Soc. London Ser. A, 104, (1923), pp: 640-654. http://dx.doi.org/10.1098/rspa.1923.0132.

[5] Takeuchi, M., "Vertical Profiles and horizontal increase of drift-snow transport", J. Glaciol, 26, (1980), pp: 481.

[6] Koch, W., "A solution of the two-dimensional atmospheric diffusion equation with height-dependent diffusion coefficient and heightdependent wind speed and eddy diffusivities", Atmospheric Environment, 23, (1989), pp: 1729-1732. http://dx.doi.org/10.1016/00046981(89)90057-7.

[7] Kevin B.M., Howard R.B. and Ronald G.R., "Numerical simulation of smoke plumes from large oil fires", Atmospheric Environment, 30, (1996), pp: 4125-4136. http://dx.doi.org/10.1016/1352-2310(96)00151-3.

[8] Bagnold, R.A., "The physics of Blown sand and Desert Dunes", Methuen, London, (1954).

[9] Kennedy, J.F., "The formation of sediment ripples, dunes and anti-dunes", Ann. Rev. Fluid Mech., 1, (1969), pp: 147-168. http://dx.doi.org/10.1146/annurev.fl.01.010169.001051.

[10] Abbott, J.E. and Francis, J.R.D., "Saltation and suspension trajectories of solid grains in a water stream", Phil. Trans. R. Soc. London, A284, (1977), pp: 225-253. http://dx.doi.org/10.1098/rsta.1977.0009.

[11] Noubissié, S. and Woafo, P., "Statistical Mechanics and its Applications", Physica A, 345, (2005), pp: 9-16. http://dx.doi.org/10.1016/j.physa.2004.06.157.

[12] Eltayeb, I.A. and Hassan, M.H.A., "On the non-linear evolution of sand dunes", Geophys. J. R. astr. Soc., 65, (1981), pp: 31-45.

[13] Schmidt, R.A., "Vertical profiles of wind speed, snow concentration, and humidity in blowing snow", Boundary-Layer Meterol. 23, (1982), pp: 233. http://dx.doi.org/10.1007/BF00123299.

[14] Pasquill, F., "Atmospheric Diffusion", D. Van Nostrand, Princeton, (1962).

[15] Gillette, D. and Goodwin, P., "Microscale transport of sand-sized soil aggregates eroded by wind", J. Geophys. Res., 79, (1974), pp:40804084. http://dx.doi.org/10.1029/JC079i027p04080.

[16] Hassan M.H.A and Eltayeb, I.A., "Time-dependent transport of dust", J. Geophys. Res., 96, (1991a), pp: 9337-9339. http://dx.doi.org/10.1029/91JD00229.

[17] Eltayeb, I.A. and Hassan, M.H.A., "Diffusion of dust particles from a point source above ground level and a line source at ground level", Geophys. J. Int. 142, (2000), pp: 426-438. http://dx.doi.org/10.1046/j.1365-246x.2000.00172.x.

[18] Zwillinger, D., "Handbook of Differential Equations", Mass, Academic Press, Boston, (1989).

[19] Abramowitz, M. and Stegun, I., "Handbook of Mathematical Functions, with Formulas, Graphs and Mathematical Tables", Dover, New York, (1965). 